\title{
Inclusive and diffractive DIS at low $x$ from HERA to the EIC
}

\author{
Konrad Tywoniuk ${ }^{* \dagger}$ \\ Departamento de Física de Partículas, \\ Universidade de Santiago de Compostela, \\ 15706 Santiago de Compostela, Spain \\ E-mail: konrad.tywoniuk@gmail.com
}

The topic of the paper is twofold. First, we describe a model for low $x$ DIS off nucleons and nuclei and discuss it's predictions for several DIS observables. Secondly, we discuss the inherent uncertainties related to extracting the structure function of the nucleus from the reduced inclusive DIS cross section with no a priori knowledge of the the nuclear effects on the parton distribution functions.

XVIII International Workshop on Deep-Inelastic Scattering and Related Subjects April 19 -23, 2010

Convitto della Calza, Firenze, Italy

\footnotetext{
* Speaker.

${ }^{\dagger}$ Work in collaboration with N. Armesto, A. B. Kaidalov, H. Paukkunen and C. A. Salgado.
} 


\section{A unitarized model for low $x$ DIS off nucleon and nuclei}

The Regge limit of QCD $\left(s \rightarrow \infty\right.$ at constant $\left.Q^{2}\right)$ is of great interest both from the perspective of high-energy collider experiments and inasmuch as it poses a challenge for the theoretical understanding of the hadronic wave-function. The rapid growth of cross sections observed at high $Q^{2}$ and fairly low $x$ is expected to slow down due to unitarity as $x \rightarrow 0$. Compared to the nucleon case, in $\gamma^{*} A$ collisions these effects are enhanced by the nuclear thickness factor $\sim A^{1 / 3}$ causing a depletion of the nuclear structure function observed at $x<0.1$, compared to the incoherent superposition of $A \gamma^{*} p$ cross sections, called nuclear shadowing

So far, all data from DIS experiments off both nucleons and nuclei can be well described by perturbative QCD (pQCD) with universal parton distribution functions (PDFs) incorporating scaling violations. But in spite of its success, this scheme has no predictive power on the energy dependence of PDFs at a given initial $Q_{0}^{2}$ rendering them uncertain, especially for the gluon sector, in the kinematical regime away from present day experiments, particularly at low $x$. Models that aim at predicting the low- $x$ behaviour of the structure functions have to include unitarity conserving mechanisms that tame the growth of cross sections at high energies. Recently, attempts to derive these corrections within $\mathrm{pQCD}$ have made significant progress.

In a frame where the target nucleus is at rest, these effects arise due to multiple scattering of the projectile. The large probability of rescattering and, thus, a large probability of diffraction dissociation, is known to arise from large, and thus principally non-perturbative, partonic fluctuations.

A suitable framework, although not rigorously established within QCD, to treat these configurations is provided by the reggeon calculus [1], where rescattering of the projectile wave function is accounted for by including multi-reggeon exchanges. In the absence of a unified QCD approach to the entirety of $\gamma^{*} N$ and $\gamma^{*} A$ processes, this framework can serve as a useful guidance for investigating the connection between non-perturbative and perturbative aspects of DIS valid for extrapolations to extremely small momentum fractions.

\subsection{DIS off protons}

In a particular realization of these models, in the $\gamma^{*}$ wave-function, one distinguishes explicitly between a large $(L)$ and a small $(S)$ component $[2,3]$. Large-mass diffraction is included through triple-reggeon interactions. With the advent of high-energy colliders the need for low- $x$ structure functions for nucleons and nuclei at high- $Q^{2}$ have arisen. This motivated an extension of the model mentioned above [3] to the perturbative regime by the inclusion of QCD scaling violations.

In [4] we describe a prescription for extracting the initial conditions at leading order for the DGLAP equations from the non-perturbative model both for inclusive $F_{2}$ and diffraction. In the former case, this procedure does not involve new parameters. The situation for the inclusive diffractive cross section is more complex, because it involves both more complicated reggeon exchanges and additional variables in the problem. For the proper description of data in the whole $\beta$ and $x_{P}$ region we identify explicitly pomeron and reggeon contributions to diffraction. One can then invoke a supplementary factorization of variables, the so-called Regge factorization, which allows for a comprehensible QCD analysis. In the reggeon case, important for small-mass diffraction, the diagrams not taken into account in the original formulation are included by a pion PDF. 


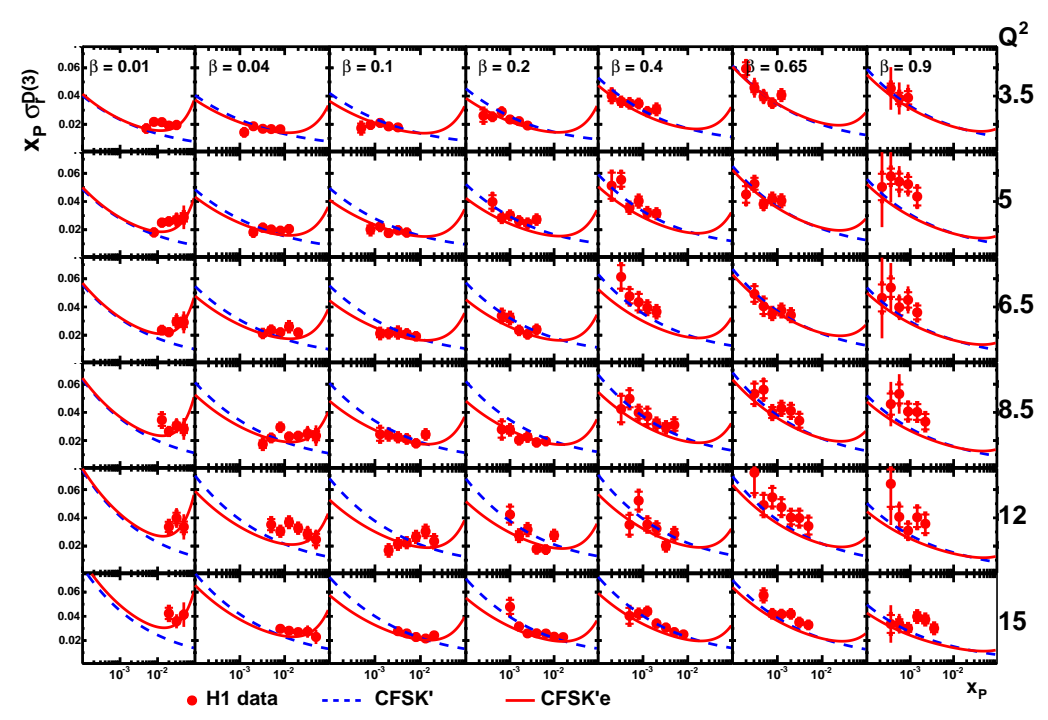

Figure 1: The proton diffractive structure function obtained from the CFSK model with QCD evolution at LO compared to a set of data from the $\mathrm{H} 1$ experiment (see [4] for details).

Thus, equipped with properly unitarized initial conditions for the DGLAP evolution equations we obtain leading-order structure functions and PDFs for the proton down to $x \sim 10^{-8}$ at high- $Q^{2}$. The resulting $F_{2}$ and $x_{P} F_{2 \mathscr{D}}^{(3)}$ are shown to be in good agreement with the most recent experimental data in Fig. 1. We have also computed the longitudinal structure function within the dipole model using the perturbative gluon PDF thus obtained. Comparisons made with the recently computed solution of the running-coupling BK equation [5] show a large discrepancy of the predicted $F_{L}$ behaviour at low $Q^{2}[4]$.

\subsection{DIS off nuclei}

In the context of DIS off nuclei, there emerges a critical length scale related to a change of the underlying space-time picture of the collision. The coherence length (or life-time) of a given fluctuation of the incoming projectile is given by

$$
l_{C}=\frac{1}{Q} \frac{E_{L A B}}{Q} \simeq \frac{1}{2 m_{N} x}
$$

in the limit $2 m_{N} E_{L A B}=W^{2} \gg Q^{2}$, where $x$ is the Bjorken variable. At low energies, where $l_{C}$ is of the order of the internucleon distance, the projectile undergoes incoherent multiple scattering off the target. Remarkably, all higher-order rescatterings cancel and the total $\gamma^{*} A$ cross section is simply given as a superposition of $\gamma^{*} p$ collisions. The critical value is reached when the coherence length becomes of the order of the nuclear radius. For $l_{C}>R_{A}$, i.e. at $x<1 / 2 m_{N} R_{A}$, the projectile scatters coherently off all constituents of the nucleus at some given impact parameter. Despite the non-local nature of the interactions, the total cross section can be written in the form of a multiple scattering series, now including corrections from higher-order rescattering diagrams which lead to an overall depletion of the total cross section, called Gribov inelastic shadowing. This formalism relates the inclusive and diffractive $\gamma^{*} p$ cross sections to the corresponding $\gamma^{*} A$ ones by means 

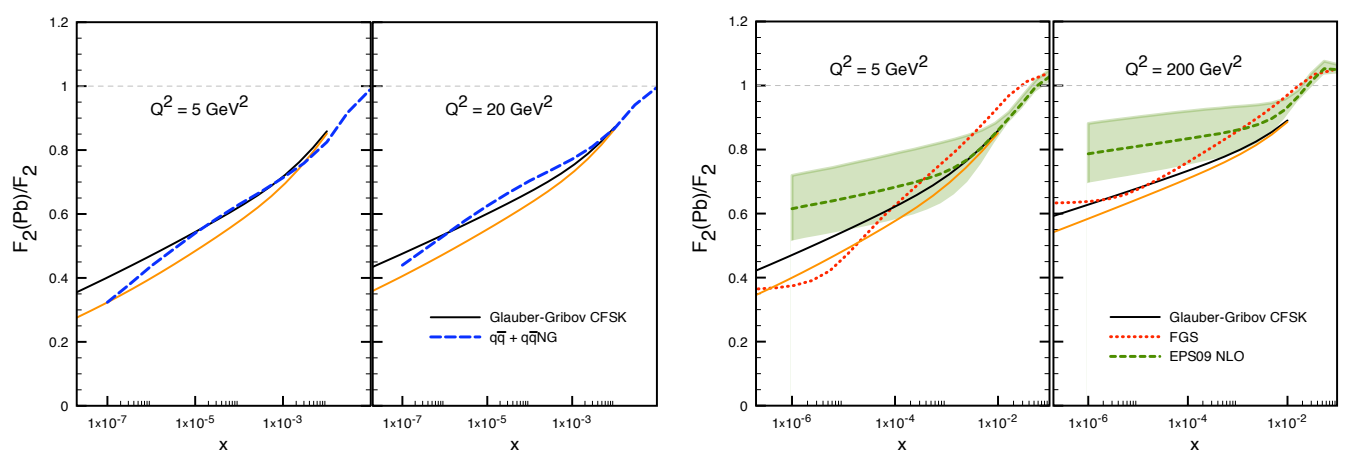

Figure 2: Results for $F_{2}(\mathrm{~Pb}) / F_{2}$ compared to the dipole calculation in [8] (left), the EPS09 NLO parameterization [9] and the FGS model [10] (right). Solid upper (black) curves are calculated in the Schwimmer model, lower (orange) ones are calculated in the eikonal model.

of the AGK cutting rules [6]. The multiple scattering has been truncated within fan and eikonal diagram re-summations. Thus our calculations for nuclei, based on the model presented in the previous subsection [4] can be extrapolated down to very low $x$ and thanks to the inclusion of scaling violations coming from QCD evolution it can also be used at high $Q^{2}$ [7]. Extension of the model to higher $\mathrm{x}$ (and low-mass diffraction) is under way.

We show the results of our calculations for $F_{2}^{\mathrm{Pb}} / F_{2}$ in Fig. 2, where also a comparison to a recent dipole model calculation [8] (right) and a NLO pQCD fit [9] together with a similar calculation to ours [10] (left) is presented. At low $x$, a large deviation from the $\mathrm{pQCD}$ parameterization is apparent. Additionally, in [7] we have also calculated high-mass diffraction off nuclei, noting that Regge factorization is broken in this case due to the large rescattering.

\section{Uncertainties on the extraction of $F_{2}^{A}$ at a future electron-ion collider}

Finally, we report on a recent study done in [11] related to the extraction of the nuclear structure function $F_{2}^{A}$ from the measured reduced cross section

$$
\sigma_{r}^{N C}=\frac{Q^{4} x}{2 \pi \alpha^{2} Y_{+}} \frac{d^{2} \sigma^{N C}}{d x d Q^{2}}=F_{2}\left[1-\frac{y^{2}}{Y_{+}} \frac{F_{L}}{F_{2}}\right],
$$

where $\alpha$ is the electromagnetic coupling constant and $Y_{+}=1+(1-y)^{2}$, stemming from the $a$ priori lack of knowledge of the expected modifications on the nuclear PDFs. Since $F_{L}^{A}$ is mainly dominated by the largely unconstrained nuclear gluons, the ratio $F_{L}^{A} / F_{2}^{A}$ can vary significantly. In order to estimate this uncertainty, we define the relative uncertainty

$$
\Delta F_{2}^{A}=\frac{\tilde{F}_{2}^{A}-F_{2}^{A}}{\tilde{F}_{2}^{A}}=1-\frac{\Delta^{p}}{\Delta^{A}},
$$

with

$$
\Delta^{p, A}=1-\frac{y^{2}}{Y_{+}} \frac{F_{L}^{p, A}}{F_{2}^{p, A}}
$$




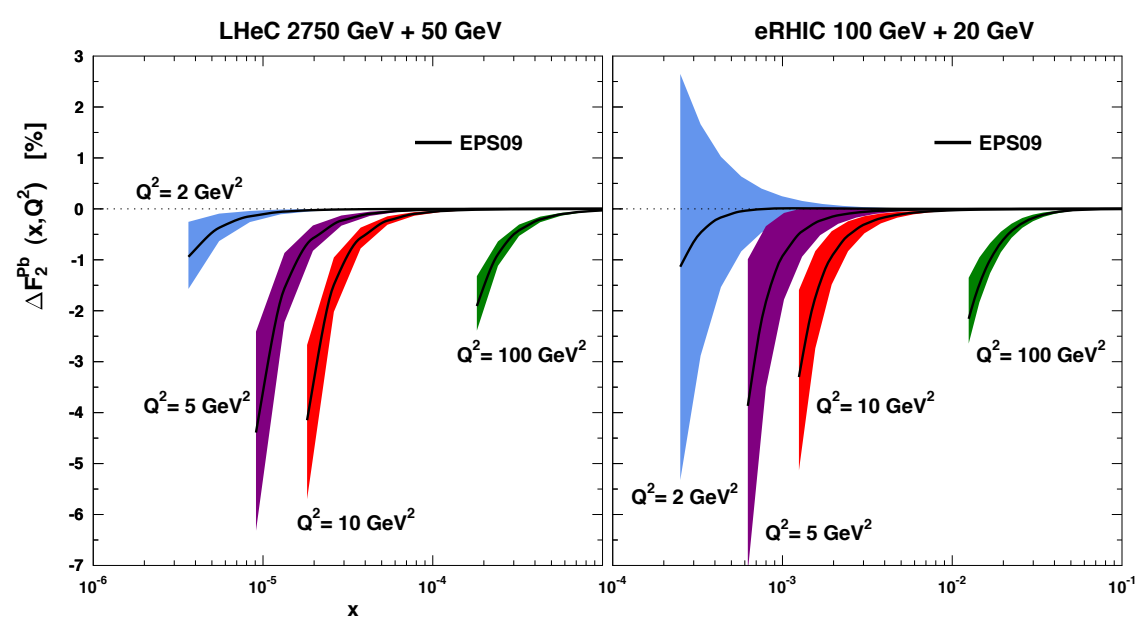

Figure 3: Results for the uncertainty in the extraction of $F_{2}$ in EPS09 [9] for two kinematics relevant for planned future electron-ion colliders.

where $\tilde{F}_{2}^{A}$ is the nuclear structure function extracted under the assumption of no nuclear effects on $F_{L} / F_{2}$, while $F_{2}^{A}$ is defined by Eq. (2.1).

We show the results for $\mathrm{Pb}$ in Fig. 3 using the parameterization of nPDFs from [9] (the uncertainty bands corresponds the uncertainties of the nPDFs). We consider two kinematical situations, a $100 \mathrm{AGeV}$ proton or nucleus on a $20 \mathrm{GeV}$ electron (left), and a $2750 \mathrm{AGeV}$ proton or nucleus on a $50 \mathrm{GeV}$ electron (right). The uncertainties introduced by the nuclear effects are sizable, rising up to $\sim 7 \%$, above all for small to moderate $Q^{2}$ and small $x$. This stresses the need of either measuring the longitudinal structure functions for nuclei or providing experimental results for the full DIS cross section in future experimental programs on lepton-nucleus collisions.

\section{References}

[1] V. N. Gribov, Sov. Phys. JETP 26 (1968) 414 [Zh. Eksp. Teor. Fiz. 53 (1967) 654].

[2] A. Capella, E. G. Ferreiro, C. A. Salgado and A. B. Kaidalov, Nucl. Phys. B 593, 336 (2001)

[3] A. Capella, E. G. Ferreiro, C. A. Salgado and A. B. Kaidalov, Phys. Rev. D 63, 054010 (2001)

[4] N. Armesto, A. B. Kaidalov, C. A. Salgado and K. Tywoniuk, Phys. Rev. D 81 (2010) 074002

[5] J. L. Albacete, N. Armesto, J. G. Milhano and C. A. Salgado, Phys. Rev. D 80, 034031 (2009)

[6] V. A. Abramovsky, V. N. Gribov and O. V. Kancheli, Yad. Fiz. 18 (1973) 595 [Sov. J. Nucl. Phys. 18 (1974) 308].

[7] N. Armesto, A. B. Kaidalov, C. A. Salgado and K. Tywoniuk, arXiv:1003.2947 [hep-ph].

[8] B. Z. Kopeliovich, J. Nemchik, I. K. Potashnikova and I. Schmidt, J. Phys. G 35 (2008) 115010

[9] K. J. Eskola, H. Paukkunen and C. A. Salgado, JHEP 0904 (2009) 065

[10] L. Frankfurt, V. Guzey and M. Strikman, Phys. Rev. D 71 (2005) 054001

[11] N. Armesto, H. Paukkunen, C. A. Salgado and K. Tywoniuk, arXiv:1005.2035 [hep-ph]. 